\title{
Endogenous BDNF is required for long-term memory formation in the rat parietal cortex
}

\author{
Mariana Alonso, ${ }^{1}$ Pedro Bekinschtein, ${ }^{2}$ Martín Cammarota, ${ }^{2}$ Monica R.M. Vianna, ${ }^{3}$ \\ Iván Izquierdo, ${ }^{4}$ and Jorge H. Medina ${ }^{2,5}$ \\ ${ }^{1}$ Department of Neuroscience, Perception and Memory Laboratory, Pasteur Institute, Paris, France; ${ }^{2}$ Instituto de Biología Celular \\ y Neurociencias, Facultad de Medicina, UBA, Buenos Aires, Argentina; ${ }^{3}$ Departamento de Bioquimica, Instituto de Biociencias, \\ UFRGS, Porto Alegre, Brazil; ${ }^{4}$ Centro de Memoria, Instituto de Pesquisas Biomedicas, PUCRS, Porto Alegre, Brazil
}

\begin{abstract}
Information storage in the brain is a temporally graded process involving different memory phases as well as different structures in the mammalian brain. Cortical plasticity seems to be essential to store stable long-term memories, although little information is available at the moment regarding molecular and cellular events supporting memory consolidation in the neocortex. Brain-derived neurotrophic factor (BDNF) modulates both short-term synaptic function and activity-dependent synaptic plasticity in hippocampal and cortical neurons. We have recently demonstrated that endogenous BDNF in the hippocampus is involved in memory formation. Here we examined the role of BDNF in the parietal cortex (PCx) in short-term (STM) and long-term memory (LTM) formation of a one-trial fear-motivated learning task in rats. Bilateral infusions of function-blocking anti-BDNF antibody into the PCx impaired both STM and LTM retention scores and decreased the phosphorylation state of cAMP response element-binding protein (CREB). In contrast, intracortical administration of recombinant human BDNF facilitated LTM and increased CREB activation. Moreover, inhibitory avoidance training is associated with a rapid and transient increase in phospho-CREB/total CREB ratio in the PCx. Thus, our results indicate that endogenous BDNF is required for both STM and LTM formation of inhibitory avoidance learning, possibly involving CREB activation-dependent mechanisms. The present data support the idea that early sensory areas constitute important components of the networks subserving memory formation and that information processing in neocortex plays an important role in memory formation.
\end{abstract}

Cortical plasticity seems to be essential to store stable memory traces. However, little information is available at the moment regarding molecular and cellular events supporting memory consolidation in the neocortex. Evidence both in human and animal models (Scoville and Milner 1957; Zola-Morgan and Squire 1990; Kim et al. 1992; Rempel-Clower et al. 1996; Bontempi et al. 1999; Graham et al. 1999) has guided the idea that the early stages of memory processing take place at the beginning in the hippocampus and that later they relay on cortical-cortical connections (McClelland et al. 1995; Squire and Alvarez 1995; Izquierdo et al. 1997; Graham et al. 1999) or cortical-hippocampal networks (Nadel and Moscovitch 1997; McGaugh 2000; Hoffman and McNaughton 2002; Izquierdo et al. 2002).

Here we studied the role of the parietal cortex (PCx) in memory formation. Although this region plays a critical role as a center for online sensory processing in mammals, recent reports suggest that it may also act as a transient storage site for information that contributes to memory processing (Harris et al. 2002).

Our previous work has demonstrated that the PCx is required for consolidation and retrieval of one-trial inhibitory avoidance memory in rats up to 1 mo after training, when the hippocampus appears not to be necessary (Zanatta et al. 1996; Izquierdo et al. 1997; Walz et al. 1999; Barros et al. 2000; Bonini et al. 2003; Rosato et al. 2004). However, cellular and molecular mechanisms underlying the establishment of memories in this and other neocortical areas are mainly unknown.

\footnotetext{
${ }^{5}$ Corresponding author.

E-mail jmedina@fmed.uba.ar; fax 54-11-4962-5457.

Article and publication are at http://www.learnmem.org/cgi/doi/10.1101/ Im.27305.
}

Brain-derived neurotrophic factor (BDNF) is a small dimeric protein that is widely expressed in the adult mammalian brain (see Murer et al. 2001), and extensively involved in synaptic plasticity and memory processes (see Poo 2001; Tyler et al. 2002). In this context, we have recently demonstrated that BDNF in the hippocampus is involved not only in memory consolidation (Alonso et al. 2002a) but also in the activity-dependent neuronal reorganization that may underlie memory formation (Alonso et al. 2004). In addition, our results suggest that BDNF exerts its role in long-term memory (LTM) formation in the CA1 region of the hippocampus, in a time-dependent manner, via the activation of the transcription factor cAMP response element-binding protein (CREB) (Alonso et al. 2002b).

Object visual learning, in which memory is presumably stored in the inferior temporal cortex, increases BDNF expression in this structure, suggesting that it could be contributing to the neuronal circuit reorganization required for visual LTM formation in primates (Tokuyama et al. 2000). More recently, Klintsova and coworkers (Klintsova et al. 2004) showed that both complex motor learning and physical exercise elicit changes in the level of expression of BDNF in cerebellum and motor cortex. However, studies are still lacking to evaluate the requirement of endogenous BDNF in memory formation either in the PCx or in other neocortical areas.

Therefore, the aim of the present study was to examine whether endogenous BDNF in the PCX is necessary for memory processing of one-trial inhibitory avoidance training in rats. Additionally, we determined whether this learning is associated with an increased in CREB activation and whether blockage of endogenous BDNF or infusion of exogenous BDNF is also able to modulate in vivo CREB phosphorylation in the PCx. 


\section{Results}

Although several reports demonstrate that cortical BDNF mRNA is induced during different learning tasks (see Tyler et al. 2002), they have not addressed the question of whether endogenous cortical BDNF is, indeed, necessary for LTM formation.

We therefore evaluated whether neutralizing endogenous BDNF biological activity, by delivering function-blocking antiBDNF antibodies (Rasika et al. 1999) into the PCx, blocked memory formation. Infusions into the PCx reached the interface between the primary somatosensory cortex (Par 1) and the supplementary somatosensory cortex (Par 2) (see Fig. 1), a region mainly involved in somatosensory information processing (Paxinos 1995).

Infusion of neutralizing BDNF antibodies $(0.5 \mu \mathrm{g} / \mathrm{side})$ into the PCx immediately after training did not affect short-term memory (STM) retention, as determined in a test session carried out $1.5 \mathrm{~h}$ later (Fig. 2A). However, when memory retention was evaluated $24 \mathrm{~h}$ after training, we found that neutralization of endogenous BDNF totally blocked LTM formation. Infusion of a control IgG $(2.5 \mu \mathrm{g} /$ side $)$ produced no effect on STM retention (test session latency $=17.5[14.13 / 29.05], n=7, p=0.1649$ with respect to saline group; Mann-Whitney $U$ test), indicating that the observed amnesia was not the result of a nonspecific proteinloading effect. Infusion of recombinant human BDNF $(0.25 \mu \mathrm{g} /$ side) into the PCx immediately after training strongly facilitated LTM retention without altering STM (Fig. 2A), supporting the idea that endogenous BDNF is required immediately after training in the PCx to form an enduring LTM.

We also found that BDNF antibody produced a full amnesia for both STM and LTM when infused $1 \mathrm{~h}$ after training. This suggests that endogenous BDNF could be acting not only on LTM processing but also on mechanisms involved in STM (Fig. 2B). No effect was observed on LTM when exogenous BDNF was administered $1 \mathrm{~h}$ or $6 \mathrm{~h}$ post-training (Fig. 2B,C).

Anti-BDNF antibody had no effect on LTM retention when given into the PCx $6 \mathrm{~h}$ after training, indicating that the amnesia produced by this antibody is not due to a deleterious effect on

A

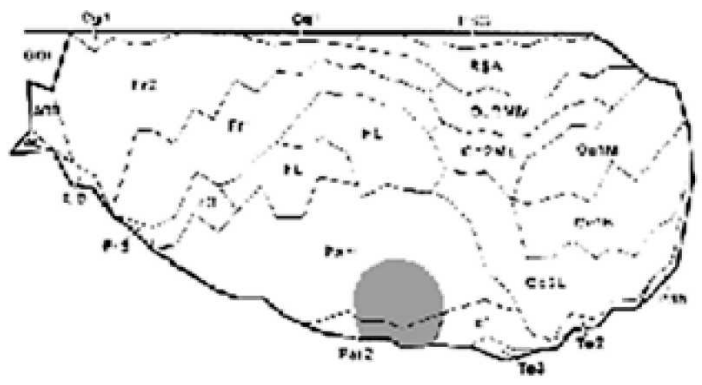

B

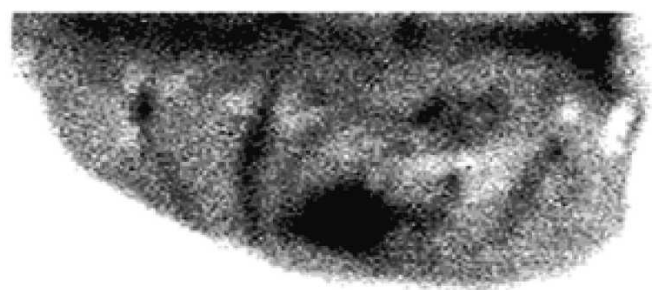

Figure 1. (A) Schematic drawing showing a dorsal view of the rat cortex in stereotaxic coordinates prepared from coronal and horizontal sections by orthogonal projection (Zilles 1985). The extent of the area reached by the infusion in the PCX (stippled) is illustrated. (Par 1) Primary somatosensory cortex; (Par 2) supplementary somatosensory cortex. (B) Representative brain with methylene blue stain depicting correct infusion placement.
A

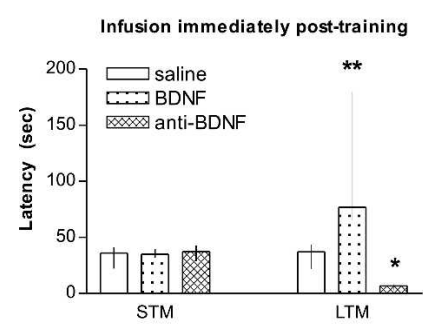

C

B
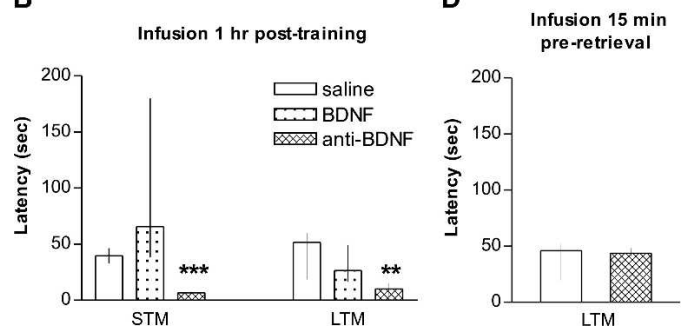

Figure 2. Cortical BDNF is required for memory formation. Medians (interquartile range) of latencies to step down from the platform of the inhibitory avoidance box in the test session $1.5 \mathrm{~h}$ (STM) and $24 \mathrm{~h}$ (LTM) after training. Animals were infused with saline, BDNF $(0.25 \mu \mathrm{g} / \mathrm{side})$, or anti-BDNF $(0.5 \mu \mathrm{g} / \mathrm{side})(A)$ immediately after avoidance training, (B) $1 \mathrm{~h}$ after training, (C) $6 \mathrm{~h}$ after training, or $(D) 15 \mathrm{~min}$ before retrieval. $\left({ }^{* *}\right) p<0.001 ;\left(^{* *}\right) p<0.01 ;\left(^{*}\right) p<0.05$ versus saline group; MannWhitney $\mathrm{U}$ test, $n=9-10$ per group.

hippocampal functionality or to a protracted action on LTM retrieval (Fig. 2C). In order to completely rule out any effect on memory retrieval, we administered anti-BDNF antibodies $15 \mathrm{~min}$ before a LTM test session $24 \mathrm{~h}$ after training. As shown in Figure 2D, no effect was seen on inhibitory avoidance memory expression.

It has been reported that BDNF may induce convulsions when infused at high doses into the hippocampus (Xu et al. 2004). At the dose we used here, neither recombinant BDNF nor the BDNF-inactivating antibody produced seizures or any other epileptic-like behavior.

In hippocampal neurons, BDNF stimulates the phosphorylation of CREB at Ser 133 (Finkbeiner et al. 1997), an event associated with the activation of this transcription factor (Montminy 1997). In this respect, we recently found that hippocampal BDNF exerts its role in LTM formation, acting, at least in part, via CREB activation (Alonso et al. 2002b).

Therefore, we decided to determine whether infusion of recombinant BDNF or anti-BDNF antibody into the PCX modifies CREB phosphorylation levels in this cortical area. Using an antibody that specifically detects CREB when phosphorylated at Ser 133 (pCREB) and an antibody that recognizes CREB independently of its phosphorylation state (total CREB), we found that when given at a dose that facilitated LTM retention $(0.25 \mu \mathrm{g} /$ side), recombinant BDNF increased the $\mathrm{PCREB} /$ total CREB ratio (Fig. 3A). Conversely, infusion of anti-BDNF antibody $(0.5 \mu \mathrm{g} /$ side), which produces amnesia, clearly decreased the pCREB/total CREB ratio (Fig. 3A). It is important to stress here that no differences were found in total CREB levels among groups $(p=0.2244$; $n=6$; one-way ANOVA), indicating that the modifications observed were exclusively due to changes in CREB activation. In agreement with previous work using cortical slices and cell cultures (Finkbeiner et al. 1997; Pizzorusso et al. 2000), our results suggest that BDNF plays a critical role on CREB phosphorylation in vivo in the PCx.

Evidence suggests that activation of CREB is a crucial step during the consolidation of one-trial inhibitory avoidance 
A
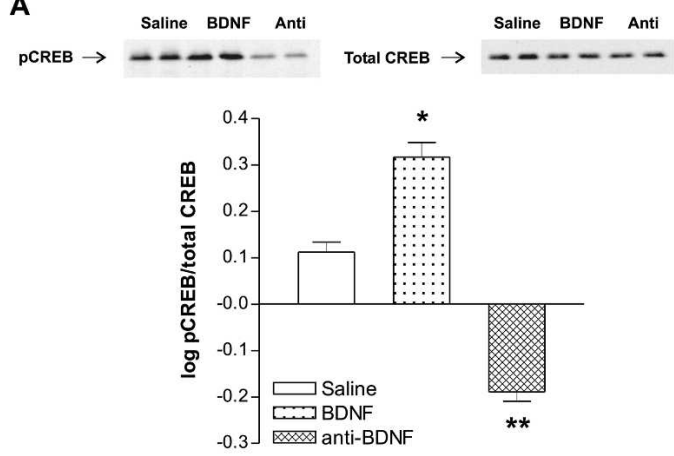

B

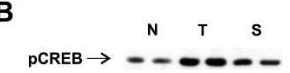

Total CREB $\rightarrow-$

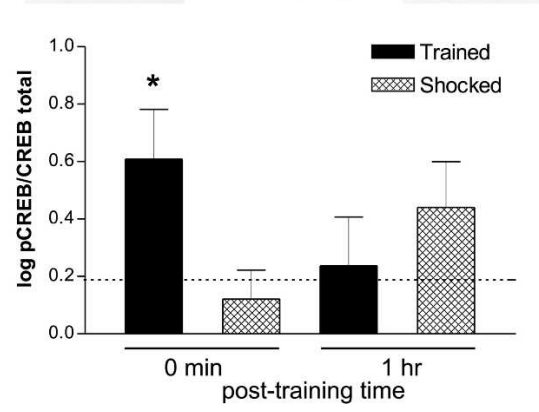

Figure 3. Inhibitory avoidance training is associated with CREB activation in the PCX. (A) Representative Western blots and densitometric analysis of the data using anti-activated CREB (PCREB) and total CREB in the nuclear fraction obtained from the area of the $P C x$ in which the infusion cannulae were placed, 30 min after animals were injected with saline or BDNF $(0.25 \mu \mathrm{g} / \mathrm{side})$ or anti-BDNF $(0.5 \mu \mathrm{g} / \mathrm{side})$. Data are expressed as the mean \pm SEM of the logarithm of the pCREB/total CREB ratio; $\left({ }^{* *}\right) p<0.01 ;\left(^{*}\right) p<0.05$; Newman-Keuls test after ANOVA, $n=5-6$ per group. (B) Representative Western blots and densitometric analysis of the data using anti-activated CREB (pCREB) and total CREB in the nuclear fraction obtained from the PCx in trained (closed bars; T) and shocked (hatched bars; S) groups, immediately after training $(0 \mathrm{~min})$ and $1 \mathrm{~h}$ after training. Data are expressed as the mean \pm SEM of the logarithm of the pCREB/total CREB ratio; $\left(^{*}\right) p<0.05$; Newman-Keuls test after ANOVA, $n=5$ per group. The dotted line represents the mean naive value $(0.1893 \pm 0.1398)$. (N) Naive group.

memory in rats (Bourtchuladze et al. 1994; Bernabeu et al. 1997; Taubenfeld et al. 1999; Cammarota et al. 2000; Viola et al. 2000). Therefore, we evaluated whether avoidance learning is also associated with changes in CREB activation in the PCX (Fig. 3B). Trained rats showed an increase in the pCREB/total CREB ratio immediately after training with respect to control shocked animals (Fig. 3B) $(p<0.05, n=5)$.

On the other hand, no differences were found in the pCREB/ total CREB ratio between trained and shocked groups $1 \mathrm{~h}$ after training (Fig. 3B) $(p=0.2472, n=5)$. In all cases, no changes in the pCREB/total CREB ratio were found in the PCx between shocked and naive control groups $(p>0.05, n=5)$. These findings indicate that inhibitory avoidance learning is associated with a rapid and transient activation of CREB transcription factor in the PCX.

It is important to stress that shocked animals submitted to a test session $24 \mathrm{~h}$ later showed no evidence of avoidance response (i.e., rats do not learn the IA task) (Depino et al. 2004). In addition, rats submitted only to one (or up to three) mild footshock(s) like those used here do not exhibit contextual fear conditioning, as indicated by the low amount of time spent in the compartment where they previously received the shock(s) (no shock, $22.0 \% \pm 6.6 \%$ total time; one shock, $19.2 \% \pm 2.4 \%$ total time; two shocks, $20.8 \% \pm 5.8 \%$ total time; three shocks, $28.3 \pm 6.0 \%$ total time; $n=10, p=0.6363$; ANOVA). Moreover, no sign of freezing was evident in any of the shocked groups (data not shown). These results indicate that exposure to a mild footshock on its own is not enough to acquire the stepping-down-shock association and/or a contextual fear. However, we cannot rule out the possibility that some form of memory (not behaviorally expressed) could be consolidated.

In neurons, CREB phosphorylation can be attained through the activation of different signaling pathways, including those mediated by ERK1/2, PKA, and CaMKII. We have previously shown that the learning-induced, BDNF-dependent activation of CREB in area CA1 seems to be mainly mediated by the ERK1/2 signaling pathway (Alonso et al. 2002b) and that the cAMP/PKA cascade is, indeed, required in the PCX for LTM formation (Ardenghi et al. 1997). In addition, it is known that CaMKII is involved in neurotrophic-dependent activation of CREB in the dentate gyrus (Blanquet et al. 2003).

Using immunoblotting, we found that infusion of antiBDNF antibody into the PCx did not affect ERK1 $(p>0.05$ in Student's $t$-test vs. Saline-infused rats; $n=6-7$ per group), ERK2 ( $p>0.05$ in Student's $t$-test vs. Saline-infused rats; $n=6-7$ per group), or CaMKII phosphorylation ( $p>0.05$ in Student's $t$-test vs. Saline-infused rats; $n=6$ per group) or modify the levels of $\alpha$ PKAc subunit $(p>0.05$ in Student's $t$-test vs. Saline-infused rats; $n=4$ per group) in nuclear extracts obtained from this cortical region. When given into the $\mathrm{PCx}$, recombinant BDNF had no effect on ERK1 activation ( $p>0.05$ in Student's $t$-test vs. Salineinfused rats; $n=6$ per group), ERK2 ( $p>0.05$ in Student's $t$-test vs. Saline-infused rats; $n=6$ per group), or in $\alpha$ PKAc nuclear levels $(p>0.05$ in Student's $t$-test vs. Saline-infused rats; $n=4$ per group), but increased Thr 286-CaMKII phosphorylation $(p<0.005$ in Student's $t$-test vs. Saline group, $n=6)$.

\section{Discussion}

The main finding of the present study is that, in the PCx, endogenous BDNF is required for the formation of inhibitory avoidance LTM during a narrow time window beginning immediately after training. In addition, our results also suggest that this requirement might involve activation of CREB-dependent mechanisms.

This is based on three series of data. First, infusion of antiBDNF antibody into the PCx immediately or $1 \mathrm{~h}$, but not $6 \mathrm{~h}$, after training impaired LTM retention (Fig. 2A-D). The anti$\mathrm{BDNF}$ antibody did not affect memory when given right before a LTM test session. These results indicate that consolidation, but not retrieval of LTM, is affected. Second, infusion of recombinant BDNF facilitated LTM retention (Fig. 2A) and increased the pCREB/total CREB ratio in PCx nuclear samples (Fig. 3A), while anti-BDNF antibody produced amnesia and decreased CREB activation (Fig. 3A). Third, trained rats showed an increase in the pCREB/total CREB ratio immediately after training, indicating that inhibitory avoidance learning is associated with a rapid and transient activation of CREB in the parietal cortical area (Fig. 3B). Our results show that endogenous BDNF is required for memory processing in the mammalian neocortex, providing important insights into the mechanisms underlying memory consolidation in the cortex. Additionally, our findings demonstrated that endogenous BDNF could be acting not only on LTM processing but also on mechanisms involved in STM, since infusion of antiBDNF antibody into the PCX $1 \mathrm{~h}$ after training impaired STM retention (Fig. 2A,B). The fact that BDNF antibody was amnesic 
when infused $1 \mathrm{~h}$ after training, whereas BDNF itself had no effect on STM or LTM (Fig. 2B), suggests that, at least at this post-training time, there is enough-or even saturated levels ofendogenous BDNF for STM and LTM formation. Therefore, additional exogenous BDNF did not facilitate memory.

Previous reports showed that the PCx is involved in one-trial inhibitory avoidance memory consolidation and retrieval (Zanatta et al. 1996; Izquierdo et al. 1997; Walz et al. 1999; Barros et al. 2000). Surprisingly, in this structure, NMDA, AMPA, or metabotropic glutamate receptors are not necessary immediately after training for inhibitory avoidance memory formation (Izquierdo et al. 1997; Bonini et al. 2003; Rosato et al. 2004), but are just starting to play a critical role $1 \mathrm{~h}$ after training. This delayed requirement of glutamatergic transmission differs from what occurs in the hippocampus, where both NMDA and AMPA receptor function is essential for memory consolidation early after training (Zanatta et al. 1996; Bonini et al. 2003; Rosato et al. 2004). Interestingly, cholinergic muscarinic transmission is required immediately post-training in the PCx for both STM and LTM formation (Izquierdo et al. 1998b), suggesting that acetylcholine and endogenous BDNF reciprocal regulation, as demonstrated in hippocampal neurons (Knipper et al. 1994), could be necessary in this structure for LTM formation around the time of training.

Although the transcription factor CREB has been classically involved in hippocampal synaptic plasticity and in the formation of hippocampal-dependent memories (Lonze and Ginty 2002), the participation of neocortical CREB in mnemonic processing has only recently commenced to be analyzed. In this respect, it has been demonstrated that mutant mice lacking $\alpha$ and $\beta$ CREB isoforms have deficits in somatosensory cortex experience-dependent plasticity (Glazewski et al. 1999). In partial agreement with our data, it has been found that PCREB levels are increased in the somatosensory cortex and in the PCx of mice submitted to fear conditioning $45 \mathrm{~min}$ or between 3 and $6 \mathrm{~h}$ after training, respectively (Stanciu et al. 2001; Wei et al. 2002). However, in our present findings, direct evidence between learningassociated CREB activation and endogenous BDNF release is still lacking. In this context, we cannot rule out that other mechanisms, different from BDNF, could also be acting on the CREB signaling cascade during inhibitory avoidance training in the PCx.

In the present report, we also evaluated the nature of the rapid events triggered in vivo by BDNF to modulate LTM formation. Studies using cell cultures, acute and cultured microslices, and in vivo microinfusion (Finkbeiner et al. 1997; Blanquet 2000; Pizzorusso et al. 2000; Alonso et al. 2002a,b) have identified several important signaling pathways that are activated by BDNF and other members of the neurotrophic factor family. These signaling cascades are initiated by the binding of neurotrophin TRK receptors leading to autophosphorylation at specific tyrosine residues within the intracellular domains (Kaplan and Stephens 1994). The phosphorylated tyrosines serve as protein interaction sites for SHC, PLC- $\gamma$, and PI3K, the intracellular molecules that lead to the activation of three major signaling pathways (Blanquet 2000). Tyrosine phosphorylation of SHC triggers activation of the MEK/ERK1/2 cascade. The active PLC- $\gamma$ cleaves phosphatidylinositol-4,5-biphosphate to generate IP3 and DAG, which, in turn, induces the release of $\mathrm{Ca}^{2+}$ from internal stores and activates PKC (Kaplan and Stephens 1994). Release of $\mathrm{Ca}^{2+}$ from internal stores also activates CaMKII and p38MAPK in hippocampal neurons (Blanquet 2000). Finally, the putative effector of PI3K is the serine and threonine kinase Akt.

Since BDNF activates the ERK1/2 cascade and induces CREB phosphorylation in hippocampal and cortical neurons (Marsh and Palfrey 1996; Xing et al. 1996; Finkbeiner et al. 1997; Han and Holtzman 2000; Pizzorusso et al. 2000; Cavanaugh et al. 2001), a likely candidate could be ERK1/2. In addition, we recently demonstrated that BDNF-induced CREB activation in the hippocampus appears to be mediated mainly through the activation of the ERK1/2 signaling pathway (Alonso et al. 2002b). Unexpectedly, our present results suggest that ERK1/2 and PKA activation is not involved in the rapid events triggered by BDNF in vivo in the PCx.

Regarding other possible BDNF-activated signaling pathways, it has been suggested that CaMKII could be modulating the synaptic events required for memory consolidation in neocortical networks (Frankland et al. 2001). In the same way, it has been demonstrated that CaMKIV, an important modulator of synaptic plasticity and memory formation in mammals, participates in long-term potentiation in primary somatosensory cortex, also contributing to CREB transcription factor phosphorylation in this structure (Wei et al. 2002).

In this context, we found that the infusion of recombinant BDNF at a dose that facilitated LTM retention (Fig. 2A) also increased CaMKII autophosphorylation in PCx nuclear-enriched samples obtained from the PCX $(p=0.0037, n=6-7$; Student's t-test). However, when given into the PCx, anti-BDNF antibody did not modify CaMKII autophosphorylation $(p>0.05, n=6$; Student's $t$-test), which indicates that although exogenous BDNF is able to promote the activation of the CaMKII cascade, this kinase is not a physiological upstream factor controlling the BDNF-induced increase in CREB phosphorylation. Further studies will be needed to elucidate the signaling pathway involved.

Finally, it is generally accepted that the cellular processes underlying learning and memory involve both pre- and postsynaptic changes at existing synapses. Therefore, endogenous BDNF release and signaling at synapses would allow modifications affecting both pre- and post-synaptic compartments, making it an ideal candidate for mediating learning-induced changes by fine-tuning the strength of synaptic connections. In fact, BDNF has effects both on hippocampal and cortical physiology by acting pre-, post-, and perisynaptically (Poo 2001; Tyler et al. 2002). In addition, several neurotrophins also play a fundamental role in the establishment of proper neuronal networks during development. In this context, BDNF induces enduring structural changes in a manner consistent with those morphological modifications thought to underlie learning and memory in the adult mammalian brain (for review, see Tyler et al. 2002).

Genoud and co-workers (Genoud et al. 2004) showed that a certain level of BDNF is required to induce formation of synapses and spines in the adult somatosensory cortex. Their study presents the first demonstration that activity-dependent control of synapse number in the adult cerebral cortex is mediated by BDNF, supporting the view that activity-induced changes in synapse number and morphology are mediated by this neurotrophin, which provides important insights into the mechanisms underlying the structural plasticity of CNS synapses in the adult cerebral cortex (Genoud et al. 2004).

In this context, it is tempting to suggest that endogenous BDNF, which in our hands is required for inhibitory avoidance memory formation in the PCx, may induce permanent changes not only at behavioral, but also at physiological and morphological levels essential to storage of the memory trace in this structure.

Additionally, the present data support the idea that early sensory areas constitute important components of the networks subserving short- and long-term memory (Kosslyn et al. 2001). This suggests that memory should be conceived of as intimately intertwined with information processing in the cortex (for review, see Eichenbaum 1997). 
In conclusion, our results indicate that endogenous BDNF is required immediately after training for memory formation in the PCx, representing an important step in unveiling the molecular mechanisms underlying neocortical memory consolidation. They also show that the molecular processes involved in the establishment of neocortical memory traces may share some mechanisms with those implicated in memory encoding in the hippocampus.

\section{Materials and Methods}

\section{Subjects}

Male Wistar rats (2-3 mo; weight 160-250 g) from our own breeding stock were used. The animals were housed in plastic cages with water and food available ad libitum, under a 12-h light/12-h dark cycle (light on at 7:00 a.m.) at a constant temperature of $23^{\circ} \mathrm{C}$.

\section{Behavioral procedures}

Inhibitory avoidance was as follows (Bernabeu et al. 1997; Izquierdo et al. 1998a): Rats were placed on a $5-\mathrm{cm}-\mathrm{high}, 8.0-\mathrm{cm}-$ wide platform to the left of a $50.0 \times 25.0 \times 25.0-\mathrm{cm}$ yellow acrylic training apparatus, whose floor was a series of parallel $0.2-\mathrm{cm}$ caliber bronze bars spaced $1.0 \mathrm{~cm}$ apart. Latency to step down onto the grid with all four paws was measured. In the training trial, immediately after stepping down onto the grid, the animals received a $0.4-\mathrm{mA}, 3.0-\mathrm{sec}$ scrambled footshock. In the test session, performed 1.5 (STM) and $24 \mathrm{~h}$ (LTM) after training, the procedures were similar except that the footshock was omitted. Significant differences on latency to step down between training and test sessions were taken as a measure of retention. All behavioral procedures were performed between 8:00 a.m. and 1:00 p.m. Two independent experiments were performed, and the operator was blind to drug infusions.

\section{Surgery and infusion procedures}

Male Wistar rats were implanted under deep thionembutal anesthesia with 27-gauge guide cannulas aimed $1 \mathrm{~mm}$ above the parietal cortex area at the coordinates of the atlas of Paxinos and Watson (1986): anterior, -2.8; lateral, \pm 5.0 ; ventral, -3.0 . Cannulae were fixed to the skull with dental acrylic (Bernabeu et al. 1997; Izquierdo et al. 1997). After recovery from the surgery, these animals were trained in inhibitory avoidance and tested 1.5 and $24 \mathrm{~h}$ later. At the time of infusion, an infusion cannula was fitted into the guide cannula. The tip of the infusion cannula protruded $1.0 \mathrm{~mm}$ beyond the guide cannula; by this procedure the drug reaches the union surface between parietal regions I and II, in agreement with the atlas of Zilles (1985). Through the infusion cannulae rats received immediately, 1 or $6 \mathrm{~h}$ after training, or $15 \mathrm{~min}$ before testing, a bilateral administration of saline, recombinant human BDNF (0.25 $\mu \mathrm{g} / \mathrm{side}$; Promega), or functionblocking anti-BDNF antibody $(0.5 \mu \mathrm{g} /$ side; Chemicon, no. $\mathrm{AB} 1513 \mathrm{P})$. Additional groups received, immediately after training, a control IgG (2.5 $\mu \mathrm{g} / \mathrm{side}$; Sigma). All drugs were dissolved in saline. In all cases infusions had a volume of $0.8 \mu \mathrm{L}$. The entire infusion procedure took $\sim 2 \mathrm{~min}$, including $30 \mathrm{sec}$ for the infusions themselves-first on one side and then on the other-and the handling. In each case, the infusion cannula was left in place for an additional $15 \mathrm{sec}$ to avoid back-flow. Drug infusions were performed between 8:00 a.m. and 3:00 p.m.

\section{Cannulae placement}

Histological examination of cannulae placements was performed as previously described (Izquierdo et al. 1997). Briefly, $24 \mathrm{~h}$ after the end of the behavioral procedures, $0.8 \mu \mathrm{L}$ of a solution of $4 \%$ methylene blue in saline was infused as indicated above. Animals were killed by decapitation 30 min later, and the brains were stored in formalin for histological localization of the infusion sites. Infusion placements were checked first by macroscopic examination according to the atlas of Zilles (1985). Histological sections showed that the depth reached by the dye from the brain surface toward cortical layers never exceeded $1.5 \mathrm{~mm}$. Infusion placements were found to be correct (i.e., within $1.5 \mathrm{~mm}^{3}$ of the intended site) in 95\% of the animals. Only behavioral data from animals in which the cannula tips were located in the intended site and the spread of injected dye did not go beyond $3.3 \mathrm{~mm}^{3}$ from those sites were included in the final analysis (see Fig. 1).

\section{Biochemical procedures}

Animals were divided into three experimental groups: (1) animals trained in inhibitory avoidance task and killed immediately or $1 \mathrm{~h}$ after training (trained group); (2) animals placed directly on the floor of the training apparatus, where they were submitted immediately to an identical footshock but were not able to make the stepping-down-shock association (shocked group) (see Depino et al. 2004), and killed immediately or $1 \mathrm{~h}$ after the procedure; and (3) animals withdrawn from their home cages at the same time points used to sacrifice the other two groups and killed immediately (naive group). The time spent in the apparatus by the shocked group was similar to that found in trained animals. This shocked group was chosen because of the possible shock effect on cortical biochemistry; thereby we had a control group that was subjected to a similar somatosensory stimulus but was unable to learn the task. After animals were killed, brains were immediately removed and parietal cortices were dissected out, frozen, and stored at $-70^{\circ} \mathrm{C}$ until used.

\section{Immunoblot assays}

To investigate whether intracortical infusion of recombinant BDNF and/or anti-BDNF antibody affects CREB, ERK1/2, and PKA activation, 3-mm-thick slices were taken $30 \mathrm{~min}$ after the infusions from the area in which the infusion cannulae were placed. Tissue was homogenized in ice-chilled buffer $(20 \mathrm{mM}$ Tris-HCL at pH 7.4, 0.32 M sucrose, 1 mM EDTA, 1 mM EGTA, 1 mM PMSF, $10 \mu \mathrm{g} / \mathrm{mL}$ aprotinin, $15 \mu \mathrm{g} / \mathrm{mL}$ leupeptin, $50 \mathrm{mM} \mathrm{NaF}$, and $1 \mathrm{mM}$ sodium orthovanadate). To evaluate levels of phosphorylated and total CREB forms and nuclear translocation of PKA catalytic subunits, nuclear pellets were prepared from homogenates as described previously (Viola et al. 2000). To investigate whether inhibitory avoidance training affects CREB activation, dissected parietal cortices from different experimental groups were homogenized and nuclear pellets were prepared as described.

In all cases, samples of homogenates (16-30 $\mu \mathrm{g}$ of protein) or nuclear fraction $(25-50 \mu \mathrm{g})$ were subjected to SDS-PAGE $(10 \%$ gels), and immunoblots were performed as previously described (Cammarota et al. 2000; Viola et al. 2000). Membranes were incubated with the following antibodies: CREB total (1:1000; New England Biolabs), anti-activated phospho-CREB (1:1000; NEB), anti-ERK1 and ERK2 total (1:3000; NEB), anti-activated phospho-ERK1 and ERK2 (1:4000; NEB), anti- $\alpha$ PKA catalytic subunit (1:5000; Santa Cruz Biotechnology), anti-pCaMKII $\alpha$ (1:5000; Promega), $\alpha$ CaMKII (1:2000; Santa Cruz Biotechnology). Film densitometric analysis was performed by using an MCID Image Analysis System (version 5.02; Imaging Research). Western blots were developed to be linear in the range used for densitometry.

\section{Data analysis}

In the inhibitory avoidance task, a ceiling of $180 \mathrm{sec}$ was imposed on the test session values. Therefore the use of nonparametric statistics was required. We used the Kruskal-Wallis test for both STM and LTM retention, followed by the individual MannWhitney U test.

Immunoblot data were analyzed by one-way ANOVA followed by the Newman-Keuls multiple comparison test or the Student's t-test when appropriate.

\section{Acknowledgments}

This work was supported by research grants from the National Council of Scientific and Technological Research of Argentina (CONICET) and the National Agency of Scientific and Techno- 
logical Promotion of Argentina (ANPCyT); and the National Research Council of Brazil (CNPq) through the National Programme for Nuclei of Excellence (PRONEX). We thank L. Müller Igaz and P. Pereira for help with some experiments.

\section{References}

Alonso, M., Vianna, M.R.M., Depino, A.M., Mello e Souza, T., Pereira, P., Szapiro, G., Viola, H., Pitossi, F., Izquierdo, I., and Medina, J.H. 2002a. BDNF-triggered events in the rat hippocampus are required for both short and long-term memory formation. Hippocampus 12: $551-560$.

Alonso, M., Vianna, M.R.M., Izquierdo, I., and Medina, J.H. 2002b. Signaling mechanisms mediating BDNF modulation of memory formation in vivo in the hippocampus. Cell. Mol. Neurobiol. 22: 647-658.

Alonso, M., Medina, J.H., and Pozzo-Miller, L. 2004. BDNF increases dendritic spine density in hippocampal CA1 pyramidal neurons via ERK1/2 activation. Learn. Mem. 11: 172-178.

Ardenghi, P., Barros, D., Izquierdo, L.A., Bevilaqua, L., Schroder, N. Quevedo, J., Rodrigues, C., Madruga, M., Medina, J.H., and Izquierdo, I. 1997. Late and prolonged post-training memory modulation in entorhinal and parietal cortex by drugs acting on the cAMP/protein kinase A signalling pathway. Behav. Pharmacol. 8: $745-751$.

Barros, D.M., Izquierdo, L.A., Mello e Souza, T., Ardenghi, P.G., Pereira, P., Medina, J.H., and Izquierdo, I. 2000. Molecular signalling pathways in the cerebral cortex are required for retrieval of one-trial avoidance learning in rats. Behav. Brain Res. 114: 183-192.

Bernabeu, R., Bevilaqua, L., Ardenghi, P., Bromberg, E., Schmitz, P., Bianchin, M., Izquierdo, I., and Medina, J.H. 1997. Involvement of hippocampal cAMP/cAMP-dependent protein kinase signaling pathways in a late memory consolidation phase of aversively motivated learning in rats. Proc. Natl. Acad. Sci. 94: 7041-7046.

Blanquet, P.R. 2000. Identification of two persistently activated neurotrophin-regulated pathways in rat hippocampus. Neuroscience 95: 705-719.

Blanquet, P.R., Mariani, J., and Derer, P. 2003. A calcium/calmodulin kinase pathway connects brain-derived neurotrophic factor to the cyclic AMP-responsive transcription factor in the rat hippocampus. Neuroscience 118: 477-490.

Bonini, J.S., Rodrigues, L., Kerr, D.S., Bevilaqua, L.R., Cammarota, M. and Izquierdo, I. 2003. AMPA/kainate and group-I metabotropic receptor antagonists infused into different brain areas impair memory formation of inhibitory avoidance in rats. Behav. Pharmacol. 14: $161-166$.

Bontempi, B., Laurent-Demir, C., Destrade, C., and Jaffard, R. 1999. Time-dependent reorganization of brain circuitry underlying long-term memory storage. Nature 400: 671-675.

Bourtchuladze, R., Frenguelli, B., Blendy, J., Schutz, G., and Silva, A. 1994. Deficient long-term memory in mice with a targeted mutation of the cAMP-responsive element binding protein. Cell 79: 59-68.

Cammarota, M., Bevilaqua, L.R.M., Ardenghi, P., Paratcha, G., Levi de Stein, M., Izquierdo, I., and Medina, J.H. 2000. Learning-associated activation of nuclear MAPK, CREB and Elk-1, along with Fos production, in the rat hippocampus after a one-trial avoidance learning: Abolition by NMDA receptor blockade. Mol. Brain Res. 76: $36-46$.

Cavanaugh, J.E., Ham, J., Hetman, M., Poser, S., Yan, C., and Xia, Z. 2001. Differential regulation of mitogen-activated protein kinases ERK1/2 and ERK5 by neurotrophins, neural activity, and cAMP in neurons. J. Neurosci. 21: 434-443.

Depino, A.M., Alonso, M., Ferrari, C., del Rey, A., Besedovsky, H., Medina, J.H., and Pitossi, F. 2004. Learning modulation by endogenous hippocampal IL-1: Blockade of endogenous IL-1 facilitates memory formation. Hippocampus 14: 526-535.

Eichenbaum, H. 1997. To cortex: Thanks for the memories. Neuron 19: $481-484$

Finkbeiner, S., Tavazoie, S., Maloratsky, A., Jacobs, K., Harris, K., and Greenberg, M. 1997. CREB: A major mediator of neuronal neurotrophin responses. Neuron 19: 1031-1047.

Frankland, P.W., O'Brien, C., Ohno, M., Kirkwood, A., and Silva, A.J. 2001. $\alpha$-CaMKII-dependent plasticity in the cortex is required for permanent memory. Nature 411: 309-313.

Genoud, C., Knott, G.W., Sakata, K., Lu, B., and Welker, E. 2004. Altered synapse formation in the adult somatosensory cortex of brain-derived neurotrophic factor heterozygote mice. J. Neurosci. 24: 2394-2400.

Glazewski, S., Barth, A.L., Wallace, H., McKenna, M., Silva, A., and Fox, K. 1999. Impaired experience-dependent plasticity in barrel cortex of mice lacking the $\alpha$ and $\delta$ isoforms of CREB. Cereb. Cortex 9: 249-256.
Graham, K.S., Patterson, K., Pratt, K.H., and Hodges, J.R. 1999. Relearning and subsequent forgetting of semantic category exemplars in a case of semantic dementia. Neuropsychology 13: 359-380.

Han, B.H. and Holtzman, D.M. 2000. BDNF protects the neonatal brain from hypoxic-ischemic injury in vivo via the ERK pathway. I. Neurosci. 20: 5775-5781.

Harris, J.A., Miniussi, C., Harris, I.M., and Diamond, M.E. 2002 Transient storage of a tactile memory trace in primary somatosensory cortex. J. Neurosci. 22: 8720-8725.

Hoffman, K.L. and McNaughton, B.L. 2002. Coordinated reactivation of distributed memory traces in primate neocortex. Science 297: 2070-2073.

Izquierdo, I., Quillfeldt, J.A., Zanatta, M.S., Quevedo, J., Schaeffer, E. Schmitz, P.K., and Medina, J.H. 1997. Sequential role of hippocampus and amygdala, entorhinal cortex and parietal cortex in formation and retrieval of memory for inhibitory avoidance in rats. Eur. J. Neurosci. 9: 786-793.

Izquierdo, I., Barros, D., Izquierdo, L., Mello e Souza, T., Izquierdo, L.A., and Medina, J.H. 1998a. Mechanisms for memory types differ. Nature 393: 635-636.

Izquierdo, I., Izquierdo, L.A., Barros, D.M., Mello e Souza, T., de Souza, M.M., Quevedo, J., Rodrigues, C., Sant'Anna, M.K., Madruga, M., and Medina, J.H. 1998b. Differential involvement of cortical receptor mechanisms in working, short-term and long-term memory. Behav. Pharmacol. 9: 421-427.

Izquierdo, I., Vianna, M.R., Izquierdo, L.A., Barros, D.M., Szapiro, G., Coitinho, A.S., Muller, L., Cammarota, M., Bevilaqua, L.R., and Medina, J.H. 2002. Memory retrieval and its lasting consequences. Neurotox. Res. 4: 573-593.

Kaplan, D.R. and Stephens, R.M. 1994. Neurotrophin signal transduction by TRK receptor. I. Neurobiol. 25: 1404-1417.

Kim, J.J. and Fanselow, M.S. 1992. Modality-specific retrograde amnesia of fear. Science 256: 675-677.

Klintsova, A.Y., Dickson, E., Yoshida, R., and Greenough, W.T. 2004 Altered expression of BDNF and its high-affinity receptor TrkB in response to complex motor learning and moderate exercise. Brain Res. 1028: 92-104.

Knipper, M., da Penha Berzaghi, M., Blochl, A., Breer, H., Thoenen, H. and Lindholm, D. 1994. Positive feedback between acetylcholine and the neurotrophins nerve growth factor and brain-derived neurotrophic factor in the rat hippocampus. Eur. J. Neurosci. 6: 668-671.

Kosslyn, S.M., Ganis, G., and Thompson, W.L. 2001. Neural foundations of imagery. Nat. Rev. Neurosci. 2: 635-642.

Lonze, B.E and Ginty, D.D. 2002. Function and regulation of CREB family transcription factors in the nervous system. Neuron 35: 605-623.

Marsh, H.N. and Palfrey, H.C. 1996. Neurotrophin-3 and brain-derived neurotrophic factor active multiple signal transduction events but are not survival factors for hippocampal pyramidal neurons. J. Neurochem. 67: 952-963.

McClelland, J.L., McNaughton, B.L., and O'Reilly, R.C. 1995. Why there are complementary learning systems in the hippocampus and neocortex: Insights from the successes and failures of connectionist models of learning and memory. Psychol. Rev. 102: 419-457.

McGaugh, J.L. 2000. Memory-A century of consolidation. Science 287: 248-251.

Montminy, M. 1997. Transcriptional regulation by cyclic AMP. Annu. Rev. Biochem. 66: 807-822.

Murer, M.G., Yan, Q., and Raisman-Vozari, R. 2001. Brain-derived neurotrophic factor in the control human brain, and in Alzheimer's disease and Parkinson's disease. Prog. Neurobiol. 63: 71-124.

Nadel, L. and Moscovitch, M. 1997. Memory consolidation, retrograde amnesia and the hippocampal complex. Curr. Opin. Neurobiol. 7: 217-227

Paxinos, G. 1995. The rat brain. Academic Press, San Diego, CA.

Paxinos, G. and Watson, C. 1986. The rat brain in stereotaxic coordinates. Academic Press, San Diego, CA.

Pizzorusso, T., Ratto, G.M., Putignano, E., and Maffei, L. 2000. Brain-derived neurotrophic factor causes cAMP response element-binding protein phosphorylation in absence of calcium increase in slices and cultured neurons from rat visual cortex. J. Neurosci. 20: 2809-2816.

Poo, M. 2001. Neurotrophins as synaptic modulators. Nat. Rev. Neurosci. 2: 24-32.

Rasika, S., Alvarez-Buylla, A., and Nottebohm, F. 1999. BDNF mediates the effects of testosterone on the survival of new neurons in an adult brain. Neuron 22: 53-62.

Rempel-Clower, N.L., Zola, S.M., Squire, L.R., and Amaral, D.G. 1996. Three cases of enduring memory impairment after bilateral damage limited to the hippocampal formation. J. Neurosci. 16: 5233-5255. 
Rosato, J.I., Bonini, J.S., Coitinho, A.S., Vianna, M.R.M., Medina, J.H., Cammarota, M., and Izquierdo, I. 2004. Retrograde amnesia induced by drugs acting on different molecular systems. Behav. Neurosci. 118: $563-568$

Scoville, W.B. and Milner, B. 1957. Loss of recent memory after bilateral hippocampal lesions. J. Neurochem. 20: 11-21.

Squire, L.R. and Alvarez, P. 1995. Retrograde amnesia and memory consolidation: A neurobiological perspective. Curr. Opin. Neurobiol. 5: $169-177$.

Stanciu, M., Radulovic, J., and Spiess, J. 2001. Phosphorylated cAMP response element binding protein in the mouse brain after fear conditioning: Relationship to Fos production. Brain Res. Mol. Brain Res. 94: 15-24.

Taubenfeld, S.M., Wiig, K.A., Bear, M.F., and Alberini, C.M. 1999. A molecular correlate of memory and amnesia in the hippocampus. Nat. Neurosci. 2: 309-310.

Tokuyama, W., Okuna, H., Hashimoto, T., Li, Y.H., and Miyashita, Y. 2000. BDNF upregulation during declarative memory formation in monkey inferior temporal cortex. Nat. Neurosci. 3: 1134-1142.

Tyler, W.J., Alonso, M., Bramham, C.R., and Pozzo-Miller, L.D. 2002. From acquisition to consolidation: On the role of brain-derived neurotrophic factor signaling in hippocampal-dependent learning. Learn. Mem. 9: 224-237.

Viola, H., Furman, M., Izquierdo, L.A., Alonso, M., Barros, D.M., de Souza, M.M., Izquierdo, I., and Medina, J.H. 2000. Phosphorylated cAMP response element-binding protein as a molecular marker of memory processing in rat hippocampus: Effect of novelty. J. Neurosci. 20: RC112 (1-5)

Walz, R., Roesler, R., Quevedo, J., Sant'Anna, M.K., Madruga, M.,
Rodrigues, C., Gottfried, C., Medina, J.H., and Izquierdo, I. 1999. Time-dependent impairment of inhibitory avoidance retention in rats by posttraining infusion of a mitogen-activated protein kinase kinase inhibitor into cortical and limbic structures. Neurobiol. Learn. Mem. 73: 11-20.

Wei, F., Qiu, C.S., Liauw, J., Robinson, D.A., Ho, N., Chatila, T., and Zhuo, M. 2002. Calcium calmodulin-dependent protein kinase IV is required for fear memory. Nat. Neurosci. 5: 573-579.

Xing, J., Ginty, D.D., and Greenberg, M.E. 1996. Coupling of the RAS-MAPK pathway to gene activation by RSK2, a growth factor-regulated CREB kinase. Science 273: 959-963.

Xu, B., Michalski, B., Racine, R.J., and Fahnestock, M. 2004. The effects of brain-derived neurotrophic factor (BDNF) administration on kindling induction, Trk expression and seizure-related morphological changes. Neuroscience 126: 521-531.

Zanatta, M.S., Schaeffer, E., Schmitz, P.K., Medina, J.H., Quevedo, J., Quillfeldt, J.A., and Izquierdo, I. 1996. Sequential involvement of NMDA receptor-dependent processes in hippocampus, amygdala, entorhinal cortex and parietal cortex in memory processing. Behav. Pharmacol. 7: 341-345.

Zilles, K. 1985. The cortex of the rat: A stereotaxic atlas. Springer-Verlag, Berlin, Heidelberg.

Zola-Morgan, S.M. and Squire, L.R. 1990. The primate hippocampal formation: Evidence for a time-limited role in memory storage. Science 250: $288-290$.

Received November 15, 2004; accepted in revised form August 8, 2005. 


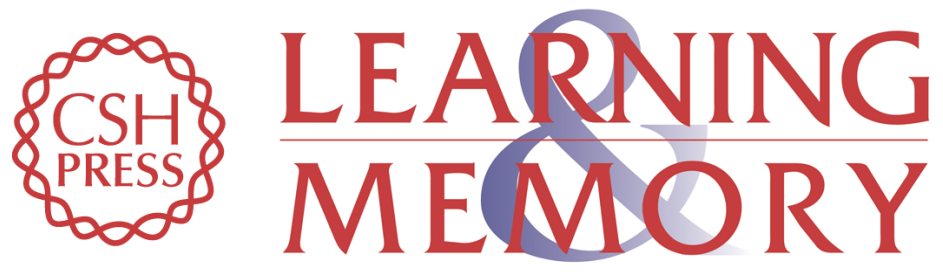

\section{Endogenous BDNF is required for long-term memory formation in the rat parietal cortex}

Mariana Alonso, Pedro Bekinschtein, Martín Cammarota, et al.

Learn. Mem. 2005, 12:

Access the most recent version at doi:10.1101/lm.27305

References This article cites 55 articles, 14 of which can be accessed free at:

http://learnmem.cshlp.org/content/12/5/504.full.html\#ref-list-1

License

Email Alerting Receive free email alerts when new articles cite this article - sign up in the box at the Service top right corner of the article or click here. 